Giancarlo Luis Gómez Gonzáles

Medição de deformações elastoplásticas em regiões de concentração de tensões utilizando métodos sem malha e visão computacional

Tese de Doutorado

Tese apresentada como requisito parcial para obtenção do título de Doutor pelo Programa de Pós-Graduação em Engenharia Mecânica do Departamento de Engenharia Mecânica da PUC-Rio.

Orientador: Prof. Marco Antonio Meggiolaro 
Giancarlo Luis Gómez Gonzáles

\section{Medição de deformações elastoplásticas em regiões de concentração de tensões utilizando métodos sem malha e visão computacional}

Tese apresentada como requisito parcial para obtenção do título de Doutor pelo Programa de Pós-Graduação em Engenharia Mecânica do Departamento de Engenharia Mecânica do Centro Técnico Científico da PUC-Rio. Aprovada pela Comissão Examinadora abaixo assinada.

Prof. Marco Antonio Meggiolaro Orientador Departamento de Engenharia Mecânica - PUC-Rio

Prof. Jaime Tupiassú Pinho de Castro Departamento de Engenharia Mecânica - PUC-Rio

Prof. José Luiz de França Freire Departamento de Engenharia Mecânica - PUC-Rio

Prof. Carlos Alberto de Almeida Departamento de Engenharia Mecânica - PUC-Rio

Prof. Luiz Carlos da Silva Nunes Departamento de Engenharia Mecânica - UFF-Rio

Prof. José Eduardo de Almeida Maneschy Eletrobrás - Eletronuclear

Prof. José Eugenio Leal Coordenador Setorial do Centro Técnico Científico - PUC-Rio Rio de Janeiro, 10 de setembro de 2014 
Todos os direitos reservados. É proibida a reprodução total ou parcial do trabalho sem autorização da universidade, do autor e do orientador.

\section{Giancarlo Luis Gómez Gonzáles}

Possui graduação em Engenharia Eletrônica pela Universidade Nacional de San Agustín de Arequipa no Perú (2006) e mestrado em Engenharia Mecânica pela Pontifícia Universidade Católica do Rio de Janeiro (2010).

Ficha Catalográfica

Gómez Gonzáles, Giancarlo Luis

Medição de Deformações Elastoplásticas em Regiões de Concentração de Tensões utilizando Métodos Sem Malha e Visão Computacional / Giancarlo Luis Gómez Gonzáles; orientador: Marco Antonio Meggiolaro 2014.

128 f. il. (color.); $30 \mathrm{~cm}$

Tese (Doutorado) - Pontifícia Universidade Católica do Rio de Janeiro, Rio de Janeiro, Departamento de Engenharia Mecânica, 2014.

Inclui bibliografia.

1. Engenharia mecânica - Teses. 2. SIFT. 3. Métodos sem malha. 4. Gradientes de deformação. 5. Deformações elastoplásticas. 6. Concentração de tensões I. Meggiolaro, Marco Antonio. II. Pontifícia Universidade Católica do Rio de Janeiro. Departamento de Engenharia Mecânica. III. Título. 
A mis queridos padres, Luis y Mariela, Que creyeron en mis sueños y ahora los ven realizados. 


\section{Agradecimentos}

Agradeço a Deus, pela inspiração.

Ao meu orientador Professor Marco Antonio Meggiolaro, pela orientação científica e conhecimentos compartilhados ao longo do mestrado e doutorado.

Aos professores membros da banca de defesa pelos pertinentes apontamentos e sugestões, contribuindo com o enriquecimento deste trabalho.

À Pontifícia Universidade Católica de Rio de Janeiro e ao Curso de PósGraduação em Engenharia Mecânica.

Ao Conselho Nacional de Desenvolvimento Científico e Tecnológico - CNPq pelo suporte financeiro no Brasil.

Aos colegas do Laboratório de Fotomecânica e Fadiga pela colaboração, apoio, conversas e amizade. 


\section{Resumo}

Gómez Gonzáles, Giancarlo Luis; Meggiolaro, Marco Antonio. Medição de deformações elastoplásticas em regiões de concentração de tensões utilizando métodos sem malha e visão computacional. PUC-Rio, 2014. 128p. Tese de Doutorado - Departamento de Engenharia Mecânica, Pontifícia Universidade Católica do Rio de Janeiro.

A análise de deformações em torno de regiões de concentração de tensões é uma importante ferramenta na avaliação da integridade estrutural de peças e componentes mecânicos. Todavia, esta análise se torna mais complexa quando o material atinge a plastificação junto ao entalhe. Neste trabalho, uma nova metodologia numérico-experimental para medição de deformações na superfície de um material, combinando métodos sem malha e visão computacional, é apresentada. A parte experimental da técnica é baseada na captura de imagens de um material em estados diferentes de deformação durante um ensaio mecânico. A técnica de visão computacional conhecida como Scale Invariant Feature Tecnique (SIFT) é utilizada aqui para extrair pontos característicos nas imagens capturadas. Para tanto, uma textura aleatória foi pintada na superfície do corpo de prova. Em seguida, os deslocamentos são obtidos experimentalmente, através do seguimento das posições dos pontos SIFT corretamente correspondidos no par de imagens capturadas do ensaio, antes e depois da aplicação da carga. Os pontos fornecidos pelo algoritmo SIFT são selecionados como nós de uma formulação sem malha, e o método de mínimos quadrados móveis é utilizado para gerar uma aproximação numérica do campo de deslocamentos e as suas derivadas. Assim, deformações na região próxima ao entalhe são devidamente quantificadas para posterior análise. $\mathrm{Na}$ validação da metodologia proposta, corpos de prova entalhados foram utilizados para estudar o comportamento da deformação plástica nas regiões de concentração de tensões. Os resultados dos testes mostraram boa concordância e precisão quando comparados com soluções analíticas, simulações pelo método dos elementos finitos (ANSYS) e soluções obtidas através de um software comercial de correlação de imagens digitais.

\section{Palavras-chave}

SIFT; métodos sem malha; gradientes de deformação; deformações elastoplásticas; concentração de tensões. 


\section{Abstract}

Gómez Gonzáles, Giancarlo Luis; Meggiolaro, Marco Antonio (Advisor). Measurement of elastoplastic strains at stress concentration regions using meshless methods and computer vision. PUC-Rio, 2014. 128p. DSc. Thesis - Departamento de Engenharia Mecânica, Pontifícia Universidade Católica do Rio de Janeiro.

Strain analysis near stress concentration regions is an important tool for structural integrity of mechanical components. However, this analysis becomes more complex when the material starts to deform plastically near the notch root. In this work, a novel experimental-numerical technique for the measurement of the strain distribution on the surface of a deformable body is described, which uses meshless methods and computer vision. The experimental part of this technique is based on the capture of images at different stages of material deformation during a mechanical test. The Scale Invariant Feature Transform (SIFT) is a computer vision algorithm used here to extract distinctive points or features in the captured images. For this purpose, a random texture was painted on the specimen surface. Then, the displacements are experimentally obtained by tracking the positions of successfully matched SIFT points in an undeformeddeformed pair of images. The points provided by SIFT are selected as nodes in a meshless formulation and the moving least square method is used to generate a numerical approximation for the displacement field and its derivates. Thus, the corresponding strain field close to the notch is calculated. To validate the proposed methodology, notched specimens were employed to study the deformation behavior on regions of stress concentration. Experimental results showed good agreement and accuracy when compared to analytical solutions, to simulations by finite elements (ANSYS) and to solutions obtained by using a commercial software based on the digital image correlation technique.

\section{Keywords}

SIFT; meshless methods; strain gradient; elastoplastic strain; stress concentration. 


\section{Sumário}

1 INTRODUÇÃO 19

1.1. Objetivos 28

1.1.1. Objetivos Específicos 28

1.2. Organização da Tese 28

2 SIFT (Scale Invariant Feature Transform) 30

2.1. Etapas do SIFT 30

2.1.1. Detecção de Extremos 30

2.1.2. Localização Precisa dos Pontos-chave 32

2.1.3. Atribuição da Orientação dos Descritores 35

2.1.4. Construção do Descritor Local 37

2.2. Matching ou Casamento de Pontos Correspondentes 38

3 ESTEREOSCOPIA

3.1. Modelo da Câmera 41

3.1.1. Parâmetros Intrínsecos 43

3.1.2. Parâmetros Extrínsecos 44

3.2. Sistema de Visão Estereoscópica 46

3.2.1. Calibração 47

3.2.2. Correspondência 48

$\begin{array}{ll}\text { 3.2.3. Reconstrução } & 48\end{array}$

4 MÉTODOS SEM MALHA 52

4.1. Princípio Básico dos Métodos Sem Malha 52

4.1.1. Conceito de Domínio de Suporte 53

4.1.2. Conceito de Domínio de Influência 54

4.2. Aproximação por Mínimos Quadrados Móveis (MLS) 55

4.2.1. Função de Base 60

4.2.2. Função Peso 61

4.3. Exemplo Numérico: Problema Unidimensional 65

4.4. Exemplo Numérico: Problema Bidimensional 67 
5 DESENVOLVIMENTO DO SISTEMA DE MEDIÇÃO VISUAL 71

5.1. Preparação do Corpo de Prova 71

5.2. Aquisição das Imagens 72

5.3. Calibração das Câmeras 73

5.4. Determinação Experimental dos Deslocamentos 75

5.5. Formulação Sem Malha 78

5.5.1. Caso № 1: Gradiente de Deformação Uniforme 79

5.5.2. Caso № 2: Gradiente de Deformação com Concentração de Tensões 86

6 RESULTADOS EXPERIMENTAIS 94

6.1. Caracterização do Material 94

6.2. Montagem Experimental 97

6.3. Análise a partir de Modelos Semi-Empíricos 99

6.3.1. Regra de Neuber 99

6.3.2. Regra de Molski e Glinka 100

6.3.3. Regra de Neuber Modificada 100

6.3.4. Regra Molski e Glinka Modificada 102

6.4. Análise por Elementos Finitos 102

6.5. Análise Utilizando o Software VIC-3D 104

6.6. Análise Utilizando o Método SIFT-Meshless 104

6.7. Comparação de Resultados 106

6.8. Análise da Incerteza nas Medições Experimentais 119

7 CONCLUSÕES E CONSIDERAÇÕES FINAIS 120

7.1. Sugestões para Trabalhos Futuros 122

8 BIBLIOGRAFIA 123 


\section{Lista de figuras}

Figura 1.1 Exemplos de regiões com concentração de tensão provocadas pela geometria da peça, com indicação dos pontos críticos (Rosa, 2002).............. 19

Figura 1.2 Variação $K_{\sigma}$ e $K_{\varepsilon}$ com a tensão no entalhe........................................... 20

Figura 1.3 Princípio da técnica DIC (Correlated, 2014)..................................... 22

Figura 1.4 Exemplo de análise utilizando o software VIC-3D............................. 24

Figura 1.5 Exemplo de correspondência utilizando o algoritmo SIFT. (a)

imagem original e (b) imagem deformada. ...................................................... 25

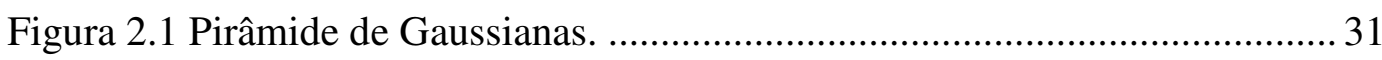

Figura 2.2 Processo de construção das imagens resultantes da Diferença de

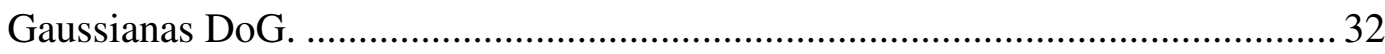

Figura 2.3 Detecção de extremos nas escalas adjacentes................................... 32

Figura 2.4 Localização precisa do ponto-chave. .................................................. 33

Figura 2.5 Exemplo de detecção de pontos-chaves. Imagem original

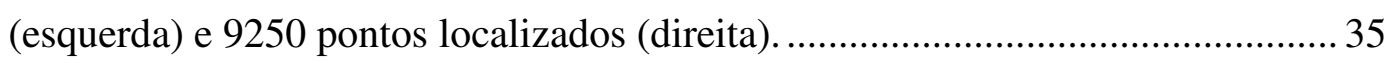

Figura 2.6 Determinação da orientação principal do ponto-chave........................ 36

Figura 2.7 Exemplo da atribuição das orientações dos pontos-chave, localizados na imagem da esquerda, representadas por vetores na imagem da

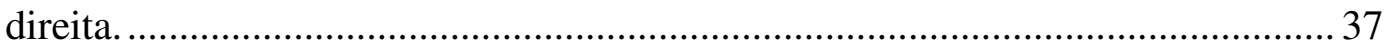

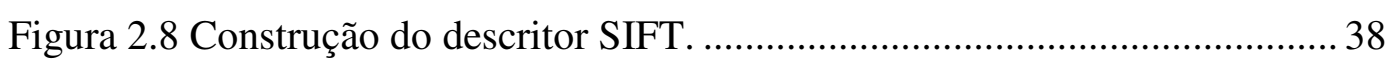

Figura 2.9 Função densidade de probabilidade típica para correspondência

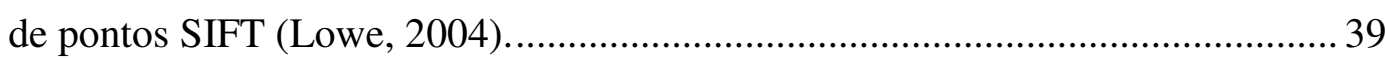

Figura 3.1 Geometria do modelo de câmera pinhole ........................................... 42

Figura 3.2 Transformação das coordenadas do mundo para as coordenadas

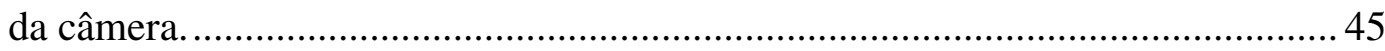

Figura 3.3 Modelo simplificado de um sistema de visão estéreo.......................... 46

Figura 3.4 Parâmetros extrínsecos e intrínsecos do sistema de visão estéreo. ...... 47

Figura 3.5 Exemplo de correspondência de pontos utilizando o algoritmo SIFT em um par de imagens capturadas simultaneamente de uma lata de refrigerante 48

Figura 3.6 Reconstrução do ponto por triangulação com raios que não se 
intersectam no espaço

Figura 3.7 Exemplo de recuperação da posição no espaço para um conjunto de pontos localizados no par-estéreo.

Figura 4.1 Representação do domínio do problema $\Omega$. Em destaque, exemplos de domínios de suporte retangular $\Omega_{i}$ e circular $\Omega_{j}$ para os nós $i$ e $j \ldots . . .53$ Figura 4.2 Exemplos de domínios de influência circulares no caso bidimensional. 54

Figura 4.3. Função de aproximação $u^{h}$ e deslocamentos nodais $u_{i}$ na aproximação MLS. 56

Figura 4.4 Exemplo de algumas funções peso no espaço unidimensional. 62

Figura 4.5 Função peso Cúbica Spline unidimensional e a sua respectiva derivada.

Figura 4.6 Função de peso Cúbica Spline bidimensional. 64

Figura 4.7 Derivada parcial da função de peso Cúbica Spline em relação à direção $x$. 64

Figura 4.8 Derivada parcial da função de peso Cúbica Spline em relação à direção $y$. 64

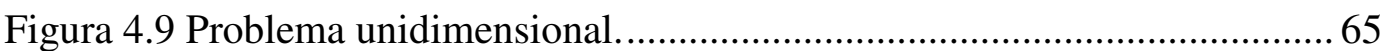

Figura 4.10 Comparação entre a solução exata e a solução MLS para $u(x)$.......... 66

Figura 4.11 Comparação entre a solução exata e a solução MLS para $u(x)_{, x}$........66

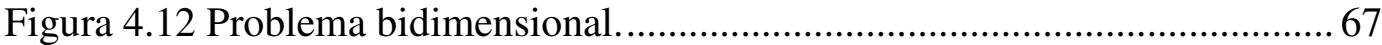

Figura 4.13 Distribuição de nós adotada na discretização do problema. 68

Figura 4.14 Comparação entre a solução exata e a solução MLS para a componente de deslocamento $u(x, y)$

Figura 4.15 Comparação entre a solução exata e a solução MLS para a componente de deslocamento $v(x, y)$

Figura 4.16 Comparação entre a solução exata e a solução MLS para a componente de deformação $\varepsilon_{x}(x, y)$

Figura 4.17 Comparação entre a solução exata e a solução MLS para a componente de deformação $\varepsilon_{y}(x, y)$.

Figura 4.18 Comparação entre a solução exata e a solução MLS para a componente de deformação $\varepsilon_{x y}(x, y)$.

Figura 5.1 Detalhe da caracterização da superfície de um corpo de prova. 
Figura 5.2 Sistema de visão estereoscópica montado no laboratório. 72

Figura 5.3 Tela principal do software Vic-Snap 2009........................................ 73

Figura 5.4 Exemplo de processo de calibração das câmeras.................................. 74

Figura 5.5 Exemplo de seleção de uma área de interesse..................................... 75

Figura 5.6 Correspondência entre cada par-estéreo............................................ 76

Figura 5.7 Correspondência entre imagem de referência e deformada................. 76

Figura 5.8 Exemplo de reconstrução da posição 3-D........................................... 78

Figura 5.9 Viga retangular submetida à flexão em quatro pontos........................ 79

Figura 5.10 Pontos correspondentes para a carga $\mathrm{P}=130 \mathrm{~N}$ (10880 pontos)....... 81

Figura 5.11 Pontos correspondentes para a carga $\mathrm{P}=190 \mathrm{~N}$ (10623 pontos) ....... 81

Figura 5.12 Evolução do erro RMS na componente $\varepsilon_{x x}$ em relação à variação do raio de influência $d_{i}$ e da constante de proporcionalidade $\alpha_{s} \ldots \ldots \ldots \ldots \ldots \ldots \ldots \ldots . . . . . . . . . . . . .82$

Figura 5.13 Evolução do erro RMS na componente $\varepsilon_{y y}$ em relação à variação

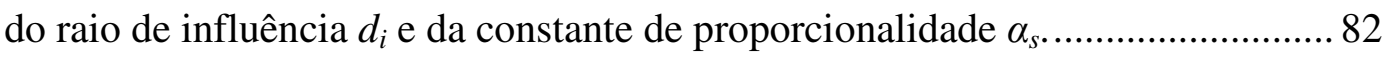
Figura 5.14 Evolução do erro RMS em relação à constante de proporcionalidade $\alpha_{s}$ na formulação sem malha para $d_{i}=2,5 \mathrm{~mm}$.

Figura 5.15 Campo de deformações na direção $x$ obtido pelo método SIFTMeshless para $\mathrm{P}=190 \mathrm{~N}$. .84

Figura 5.16 Campo de deformações na direção $y$ obtido pelo método SIFT-

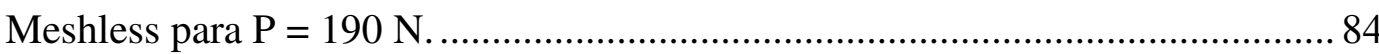

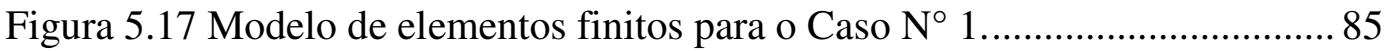

Figura 5.18 Comparação entre resultados numérico e experimental para $\varepsilon_{x x} \ldots \ldots . . .85$

Figura 5.19 Comparação entre resultados numérico e experimental para $\varepsilon_{y y} \ldots \ldots . . .85$

Figura 5.20 Viga engastada com carregamento na extremidade.......................... 86

Figura 5.21 Pontos correspondentes para $\mathrm{P}=4.7 \mathrm{~N}(10254$ pontos $)$................... 87

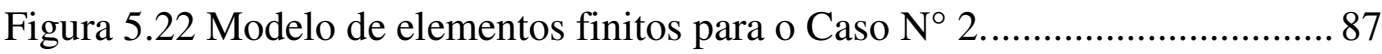

Figura 5.23 Comparação entre resultados numérico e experimental da distribuição de $\varepsilon_{x x}$ ao longo da seção transversal em $x=0$. 88

Figura 5.24 Pontos SIFT utilizados na formulação sem malha (Esq.).

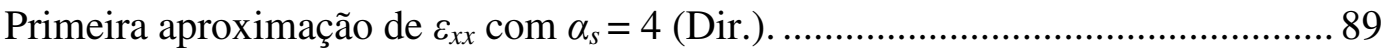

Figura 5.25 Cálculo do gradiente normalizado de $\varepsilon_{x x}$ na direção $y$. .89

Figura 5.26 Variação do gradiente normalizado calculado na direção y para as simulações com raios de entalhe de 1,5 e $10 \mathrm{~mm}$. 
Figura 5.27 Função calibrada para determinação do parâmetro $\alpha_{s}$.

90

Figura 5.28 Campo de deformações $\varepsilon_{x x}$ na direção $x$ obtido pelo método SIFT-Meshless para o Caso $\mathrm{N}^{\circ} 2$.

Figura 5.29 Comparação entre resultados numérico e experimental para a componente de deformação $\varepsilon_{x x}$ na direção $x$.

Figura 5.30 Comparação entre resultados numérico e experimental da distribuição de $\varepsilon_{x x}$ ao longo da seção transversal em $x=0$. 92

Figura 6.1 Geometria do corpo de prova utilizado para caracterização do aço 304 (dimensões em milímetros). 94

Figura 6.2 Curva $\sigma-\varepsilon$ de engenharia e real para o aço inox 304 .......................... 95

Figura 6.3 Determinação do módulo de elasticidade para o aço inox 304............ 95

Figura 6.4 Ajuste do coeficiente $H$ e do expoente $h$ para o aço 304. ................... 96

Figura 6.5 Ajuste de Ramberg-Osgood para o aço 304....................................... 96

Figura 6.6 Geometria do corpo de prova utilizado para o ensaio de flexão (dimensões em milímetros) 97

Figura 6.7 Corpo de prova utilizado no ensaio de flexão em quatro pontos 97

Figura 6.8 Viga retangular com entalhe semicircular submetida a flexão em quatro pontos. .98

Figura 6.9 Montagem experimental. 98

Figura 6.10 Discretização do modelo do corpo de prova em elementos finitos

Figura 6.11 Exemplo de configuração do modelo de encruamento em ANSYS.

Figura 6.12 Região de interesse analisada no software VIC-3D 104

Figura 6.13 Pontos correspondentes localizados na imagem de referência pelo algoritmo SIFT.

Figura 6.14 Nós no domínio do problema e domínio de influência mínimo $\left(d_{i}=2,5 \mathrm{~mm}\right)$ utilizados na formulação sem malha

Figura 6.15 Campo de deformações na direção horizontal $x$ obtido pelo método SIFT-Meshless para a carga de $4 \mathrm{kN}$.

Figura 6.16 Campo de deformações na direção horizontal $x$ obtido pelo método SIFT-Meshless para a carga de $8 \mathrm{kN}$.

Figura 6.17 Campo de deformações na direção horizontal $x$ obtido pelo 
software VIC-3D para a carga de $4 \mathrm{kN}$.

Figura 6.18 Campo de deformações na direção horizontal $x$ obtido pelo software VIC-3D para a carga de $8 \mathrm{kN}$.

Figura 6.19 Campo de deformações na direção horizontal $x$ obtido pelo software ANSYS para a carga de $4 \mathrm{kN}$.

Figura 6.20 Campo de deformações na direção horizontal $x$ obtido pelo software ANSYS para a carga de $8 \mathrm{kN}$.

Figura 6.21 Comparação entre resultados numérico e experimental para a componente de deformação na direção horizontal $x$ com carga $4 \mathrm{kN}$.

Figura 6.22 Comparação entre resultados numérico e experimental para a componente de deformação na direção horizontal $x$ com carga de $8 \mathrm{kN}$.

Figura 6.23 Comparação de resultados para deformação máxima na direção horizontal $x$ obtidos pelas regras de Neuber e Molsky-Glinka, o Método de Elementos Finitos (ANSYS), e o método SIFT-Meshless.

Figura 6.24 Comparação de resultados para deformação máxima na direção horizontal $x$ obtidos pelas regras modificadas de Neuber e Molski-Glinka, e o método SIFT-Meshless

Figura 6.25 Comparação de resultados para $K_{\varepsilon}$ obtidos pelas regras de Neuber e Molski-Glinka, regras modificadas de Neuber e Molski-Glinka, e o método de Elementos Finitos (ANSYS). 114

Figura 6.26 Distribuições de $\varepsilon_{x x}$ na posição $x=0$ para $\mathrm{P}=3 \mathrm{kN}$. 115

Figura 6.27 Distribuições de $\varepsilon_{x x}$ na posição $x=0$ para $\mathrm{P}=4 \mathrm{kN}$. 116

Figura 6.28 Distribuições de $\varepsilon_{x x}$ na posição $x=0$ para $\mathrm{P}=5 \mathrm{kN}$. 116

Figura 6.29 Distribuições de $\varepsilon_{x x}$ na posição $x=0$ para $\mathrm{P}=6 \mathrm{kN}$. 116

Figura 6.30 Distribuições de $\varepsilon_{x x}$ na posição $x=0$ para $\mathrm{P}=7 \mathrm{kN}$. 117

Figura 6.31 Distribuições de $\varepsilon_{x x}$ na posição $x=0$ para $\mathrm{P}=8 \mathrm{kN}$. 117

Figura 6.32 Distribuições de $\varepsilon_{x x}$ na posição $x=0$ para $\mathrm{P}=9 \mathrm{kN}$. 117

Figura 6.33 Distribuições de $\varepsilon_{x x}$ na posição $x=0$ para $\mathrm{P}=10 \mathrm{kN}$. 118

Figura 6.34 Distribuições de $\varepsilon_{x x}$ na posição $x=0$ para $\mathrm{P}=11 \mathrm{kN}$. 118

Figura 6.35 Distribuições de $\varepsilon_{x x}$ na posição $x=0$ para $\mathrm{P}=12 \mathrm{kN}$. 118 


\section{Lista de tabelas}

Tabela 4.1 Algumas bases polinomiais. ............................................................. 60

Tabela 4.2 Erros RMS no cálculo da solução para o problema unidimensional...66

Tabela 4.3 Erros RMS no cálculo da solução do problema bidimensional........... 70

Tabela 5.1 Detalhes do processamento das imagens pelo SIFT........................... 77

Tabela 5.2 Descrição do corpo de prova. ............................................................... 80

Tabela 5.3 Detalhes do processamento SIFT para $\mathrm{P}=130 \mathrm{~N}$............................ 80

Tabela 5.4 Detalhes do processamento SIFT para $\mathrm{P}=190 \mathrm{~N}$.............................. 80

Tabela 5.5 Erros RMS obtidos para diferentes funções peso................................ 83

Tabela 5.6 Descrição do corpo de prova ............................................................. 86

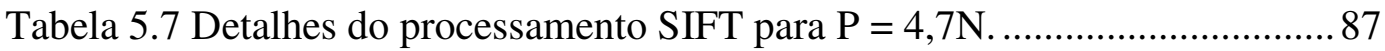

Tabela 6.1 Resultados obtidos para o cálculo do $K_{t}$ linear elástico.......................99

Tabela 6.2 Detalhes do processamento SIFT para o corpo de prova metálico.... 105

Tabela 6.3 Resultados de máxima deformação na direção horizontal $x$.............. 110

Tabela 6.4 Análise de incerteza para o método utilizado................................... 119 


\section{Abreviaturas e símbolos}

\begin{tabular}{|c|c|}
\hline ASTM & American Society for Testing and Materials \\
\hline $\mathrm{CCD}$ & Charge-coupled device \\
\hline DEM & Diffuse Element Method \\
\hline DIC & Digital Image Correlation \\
\hline DoG & Difference of Gaussians \\
\hline EFG & Element-free Galerkin \\
\hline FEM & Finite Element Method \\
\hline FDP & Função Densidade de Probabilidade \\
\hline MLS & Moving Least Square \\
\hline SIFT & Scale Invariant Feature Transform \\
\hline$a, b$ & Constantes da função peso \\
\hline$a(x)$ & Vetor de coeficientes \\
\hline$C$ & Centro de projeção \\
\hline \multirow[t]{2}{*}{$D$} & Função diferença de gaussianas, ou \\
\hline & altura da seção reta de uma viga [mm] \\
\hline Det & Determinante de uma matriz \\
\hline$d m$ & Dimensão do domínio de influência [mm] \\
\hline$d$ & Raio de influência [mm] \\
\hline$E$ & Modulo de elasticidade [GPa] \\
\hline$e_{o}$ & Erro \\
\hline$e(x)$ & Erro residual \\
\hline$f$ & Distância focal \\
\hline$F$ & Imagem filtrada \\
\hline$G$ & Módulo de elasticidade ao cisalhamento [GPa] \\
\hline \multirow[t]{2}{*}{$H$} & Matriz Hessiana, ou \\
\hline & coeficiente de encruamento monotônico [MPa] \\
\hline$h$ & expoente de encruamento monotônico \\
\hline \multirow[t]{2}{*}{$I$} & Imagem de entrada, ou \\
\hline & momento de inércia $\left[\mathrm{mm}^{3}\right]$ \\
\hline$J$ & Funcional ou função cujo domínio é um espaço vetorial \\
\hline
\end{tabular}


Constante da função peso

$k_{1}, k_{2}$

Coeficientes de distorção radial

K

Matriz de parâmetros intrínsecos da câmera

$K_{t}$

Fator de concentração de tensão linear elástico

$K_{t}^{M}$

Fator de concentração de tensão linear elástico modificado

$K_{\varepsilon}$

Fator de concentração de deformação elastoplástico

$K_{\sigma}$

Fator de concentração de tensão elastoplástico

$K_{p}$

Fator de plasticidade do entalhe

$L$

Comprimento [mm]

$L_{i}, L_{s}$

Comprimento do vão inferior e vão superior [mm]

$L_{p}$

Carga de escoamento [MPa]

$L_{y}$

Carga de colapso plástico [MPa]

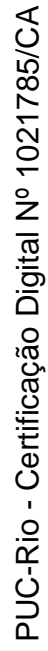

$M$

$m$

$\log , \ln$

$r$

$r_{i j}$

$R$

$R$

$S_{y}$

$u, v$

$P$

$P(x)$

$P_{e}, P_{d}$

sign

$T$

$t$

Posição de um ponto no espaço

Magnitude do gradiente do descritor

Logaritmos na base 10 e na base $e(e \approx 2.71828)$

Razão, ou

raio do entalhe [mm]

Elemento na i-ésima linha e na j-ésima coluna da matriz

de rotação

Raio da distorção radial [pixels]

Matriz de rotação

Coeficiente skew da câmera

Resistência ao escoamento [MPa]

Deslocamentos nas direções dos eixos x e y

Carga aplicada $[\mathrm{N}]$

Base polinomial

Raio de projeção esquerda e direita

Função sinal

Vetor de translação

Espessura da peça [mm] 


\begin{tabular}{|c|c|}
\hline $\operatorname{tr}$ & Traço de uma matriz \\
\hline$t_{x}, t_{y}, t_{z}$ & Componentes de do vetor de translação \\
\hline$w(x)$ & Função peso \\
\hline$\alpha$ & Autovalor com maior magnitude \\
\hline$\alpha_{s}$ & Constante de proporcionalidade \\
\hline$\beta$ & Autovalor com menor magnitude \\
\hline$\phi(x)$ & Função de forma \\
\hline$\varphi$ & Magnitude do elemento de um descritor \\
\hline$\varepsilon$ & Deformação real \\
\hline$\varepsilon_{\text {eng }}$ & Deformação de engenharia \\
\hline$\varepsilon_{m}$ & Deformação máxima \\
\hline$\varepsilon_{n}$ & Deformação nominal \\
\hline$\varepsilon_{x x}, \varepsilon_{y y}, \varepsilon_{x y}$ & Componentes do tensor deformação \\
\hline$\varepsilon_{n}^{M}$ & Deformação nominal modificada [MPa] \\
\hline$\mu \varepsilon$ & Abreviatura de "microstrain" $\left(1 \mu \varepsilon=10^{-6} \mathrm{~m} / \mathrm{m}\right)$ \\
\hline$v$ & Coeficiente de Poisson \\
\hline$\pi$ & Plano de imagem da câmera \\
\hline$\Theta$ & Descritor associado a um ponto característico \\
\hline$\theta$ & Ângulo de rotação, ou \\
\hline & orientação do gradiente do descritor \\
\hline$\sigma$ & Tensão real [MPa] \\
\hline$\sigma_{\text {eng }}$ & Tensão de engenharia [MPa] \\
\hline$\sigma_{n}$ & Tensão nominal [MPa] \\
\hline$\sigma_{m}$ & Tensão máxima [MPa] \\
\hline$\sigma_{g}$ & Desvio padrão do filtro Gaussiano \\
\hline$\sigma_{n}^{M}$ & Tensão nominal modificada [MPa] \\
\hline$\omega_{x}, \omega_{y}, \omega_{z}$ & Rotação em torno do eixo x, y e $\mathrm{z}$, respectivamente \\
\hline$\psi$ & Magnitude do elemento de um descritor \\
\hline
\end{tabular}

